\title{
Self-Dual Fields Harbored by a Kerr-Taub-bolt Instanton
}

\author{
A. N. Aliev \\ Feza Gürsey Institute, P.K. 6 Çengelköy, 81220 Istanbul, Turkey \\ Cihan Saçlıŏlu \\ Faculty of Engineering and Natural Sciences, \\ Sabanci University, 81474 Tuzla, Istanbul, Turkey
}

(Dated: October 30, 2018)

\begin{abstract}
We present a new exact solution for self-dual Abelian gauge fields living on the space of the Kerr-Taub-bolt instanton, which is a generalized example of asymptotically flat instantons with non-self-dual curvature, by constructing the corresponding square integrable harmonic form on this space.
\end{abstract}


Gravitational instantons are usually defined as complete nonsingular solutions of the vacuum Einstein field equations in Euclidean space [1], 2], [3]-[5]. Among other things, they play an important role in the path-integral formulation of quantum gravity [6], [7] forming a privileged class of stationary phase metrics that provide the dominant contribution to the path integral and mediate tunneling phenomena between topologically inequivalent vacua. The first examples of gravitational instanton metrics were obtained by complexifying the Schwarzschild, Kerr and Taub-NUT spacetimes through analytically continuing them to the Euclidean sector [1],[2]. The Euclidean Schwarzschild and Euclidean Kerr solutions do not have self-dual curvature though they are asymptotically flat at spatial infinity and periodic in imaginary time, while the Taub-NUT instanton is self-dual. However, there exists another type of Taub-NUT instanton which, unlike the first one, is not self-dual and possesses an event horizon ("bolt") 8]. The generalization of this Taub-bolt metric to the rotating case was given in [9].

Another class of gravitational instanton solutions consists of the Eguchi-Hanson metric 10] and the multi-centre metrics of [2], which include the former as a special case. These metrics are asymptotically locally Euclidean with self-dual curvature and admit a hyperKähler structure. (For a review see [1]]). The hyper-Kähler structure of gravitational instantons and some properties of gravitational instantons which are derivable from minimal surfaces in 3-dimensional Euclidean space were examined in [12], [13] using the NewmanPenrose formalism for Euclidean signature.

A fundamental difference between manifolds that have Euclidean $(++++)$ and Lorentzian $(-+++)$ signatures is that the former can harbor self-dual gauge fields that have no effect on the metric, while in the latter external fields serve as source terms in field equations. In other words, since the energy-momentum tensor vanishes identically for self-dual gauge fields, solutions of Einstein's equations automatically satisfy the system of coupled EinsteinMaxwell and Einstein-Yang-Mills equations. The corresponding self-dual gauge fields are inherent in the given instanton metric. Furthermore, in Euclidean signature, Weyl spinors also have vanishing energy-momentum tensor and vector and axial- vector bilinear covariants. Hence they cannot appear as source terms in the field equations as well. The explicit solutions for different configurations of some "stowaway" gauge fields and spinors living on well-known Euclidean-signature manifolds have been obtained in a number of papers (see [14]-[19]) . 
In recent years, motivated by Sen's $S$-duality conjecture [20], there has been some renewed interest in self-dual gauge fields living on well-known Euclidean-signature manifolds. The gauge fields were studied by constructing self-dual square integrable harmonic forms on given spaces. For instance, the square integrable harmonic 2-form in self-dual Taub-NUT metrics was constructed in [21], its generalization to the case of complete noncompact hyperKähler spaces was given in [22]. However the similar square integrable harmonic form on manifolds with non-self-dual metrics was found only for the simple case of the EuclideanSchwarzschild instanton [23]. In this note we shall give a new exact solution to describe the Abelian "stowaway" gauge fields harbored by the Kerr-Taub-bolt instanton, which is a generalized example of asymptotically flat instantons with non-self-dual curvature. This is achieved by explicit construction of the corresponding square integrable harmonic form on the space.

The Euclidean Kerr-Taub-bolt instanton was discovered by Gibbons and Perry [9] as a rotating generalization of the earlier Taub-bolt solution [8] with non-self-dual curvature. This Ricci-flat metric is still asymptotically flat and in Boyer-Lindquist type coordinates it has the form

$$
d s^{2}=\Xi\left(\frac{d r^{2}}{\Delta}+d \theta^{2}\right)+\frac{\sin ^{2} \theta}{\Xi}\left(\alpha d t+P_{r} d \varphi\right)^{2}+\frac{\Delta}{\Xi}\left(d t+P_{\theta} d \varphi\right)^{2},
$$

where the metric functions are given by

$$
\begin{aligned}
\Delta & =r^{2}-2 M r-\alpha^{2}+N^{2}, \\
\Xi & =P_{r}-\alpha P_{\theta}=r^{2}-(N+\alpha \cos \theta)^{2}, \\
P_{r} & =r^{2}-\alpha^{2}-\frac{N^{4}}{N^{2}-\alpha^{2}}, \\
P_{\theta} & =-\alpha \sin ^{2} \theta+2 N \cos \theta-\frac{\alpha N^{2}}{N^{2}-\alpha^{2}} .
\end{aligned}
$$

The parameters $M, N, \alpha$ represent the "electric" mass, "magnetic" mass and "rotation" of the instanton, respectively.

When $\alpha=0$ this metric reduces to the Taub-bolt instanton solution found in [8] with an event horizon and non-self-dual curvature. If $N=0$, we have the Euclidean Kerr metric. Thus one can say that the metric (11) generalizes the Taub-bolt solution of [8] in same manner just as the Kerr metric generalizes the Schwarzschild solution. The coordinate $t$ in the metric behaves like an angular variable and in order to have a complete nonsingular 
manifold at values of $r$ defined by equation $\Delta=0, t$ must have a period $2 \pi / \kappa$. The coordinate $\varphi$ must also be periodic with period $2 \pi(1-\Omega / \kappa)$, where the "surface gravity" $\kappa$ and the "angular velocity" of rotation $\Omega$, are defined as

$$
\kappa=\frac{r_{+}-r_{-}}{2 r_{0}^{2}}, \quad \Omega=\frac{\alpha}{r_{0}^{2}}
$$

with

$$
r_{ \pm}=M \pm \sqrt{M^{2}-N^{2}+\alpha^{2}}, \quad r_{0}^{2}=r_{+}^{2}-\alpha^{2}-\frac{N^{4}}{N^{2}-\alpha^{2}}
$$

As a result one finds that the condition

$$
\kappa=\frac{1}{4|N|}
$$

along with $\Xi \geq 0$ for $r>r_{+}$and $0 \leq \theta \leq \pi$ guarantees that $r=r_{+}$is a regular bolt in the nonsingular manifold of (11).

We shall also need the basis one-forms for the metric (11) which can be chosen as

$$
\begin{aligned}
e^{1} & =\left(\frac{\Xi}{\Delta}\right)^{1 / 2} d r, \quad e^{2}=\Xi^{1 / 2} d \theta, \\
e^{3} & =\frac{\sin \theta}{\Xi^{1 / 2}}\left(\alpha d t+P_{r} d \varphi\right), \\
e^{4} & =\left(\frac{\Delta}{\Xi}\right)^{1 / 2}\left(d t+P_{\theta} d \varphi\right) .
\end{aligned}
$$

The isometry properties of the Kerr-Taub-bolt instanton with respect to a $U(1)$ - action in imaginary time imply the existence of the Killing vector field

$$
\frac{\partial}{\partial t}=\xi_{(t)}^{\mu} \frac{\partial}{\partial x^{\mu}}
$$

We recall that the fixed point sets of this Killing vector field describe a two-surface, or bolt, in the metric. We shall use the Killing vector to construct a square integrable harmonic 2-form on the Kerr-Taub-bolt space. It is well-known that for a Ricci-flat metric a Killing vector can serve as a vector potential for associated Maxwell fields in this metric 24]. Since our Kerr-Taub-bolt instanton is also Ricci-flat, it is a good strategy to start with the Killing one-form field

$$
\xi=\xi_{(t) \mu} d x^{\mu}
$$


which is obtained by lowering the index of the Killing vector field in (9). Taking the exterior derivative of the one-form in the metric (1) we have

$$
\begin{aligned}
d \xi= & \frac{2}{\Xi^{2}}\left\{\left[M r^{2}+(\alpha M \cos \theta-2 N r+M N)(N+\alpha \cos \theta)\right] e^{1} \wedge e^{4}\right. \\
& \left.-\left[N\left(\Delta+\alpha^{2}+\alpha^{2} \cos ^{2} \theta\right)+2 \alpha\left(N^{2}-M r\right) \cos \theta\right] e^{2} \wedge e^{3}\right\} .
\end{aligned}
$$

In this expression we have used the basis one-forms (9) in order to facilitate the calculation of its Hodge dual, which is based on the simple relations

$$
\star\left(e^{1} \wedge e^{4}\right)=e^{2} \wedge e^{3}, \quad \star\left(e^{2} \wedge e^{3}\right)=e^{1} \wedge e^{4} .
$$

Straightforward calculations using the above expressions show that the two-form (11) is both closed and co-closed, that is, it is a harmonic form. However the Kerr-Taub-bolt instanton does not admit hyper-Kähler structure, and the two-form given by (11) is not self-dual. Instead, we define the (anti)self-dual two form

$$
F=\frac{\lambda}{2}\left(d \xi \pm^{\star} d \xi\right)
$$

where $\lambda$ is an arbitrary constant related to the dyon charges carried by the fields and the minus sign refers to the anti-self-dual case. Taking equations (11) and (12) into account in this expression, we obtain the harmonic self-dual two-form

$$
F=\frac{\lambda(M-N)}{\Xi^{2}}(r+N+\alpha \cos \theta)^{2}\left(e^{1} \wedge e^{4}+e^{2} \wedge e^{3}\right)
$$

which implies the existence of the potential one-form

$$
\left.A=-\lambda(M-N)\left[\cos \theta d \varphi+\frac{r+N+\alpha \cos \theta}{\Xi}\left(d t+P_{\theta} d \varphi\right)\right)\right] .
$$

After an appropriate re-scaling of the parameter $\lambda$, which includes the electric coupling constant as well, a string singularity at $\theta=0$ or $\theta=\pi$ in this expression is avoided as usual by demanding the familiar Dirac magnetic-charge quantization rule.

From equation (13) we also find the corresponding anti-self-dual two-form

$$
F=\frac{\lambda(M+N)}{\Xi^{2}}(r-N-\alpha \cos \theta)^{2}\left(e^{1} \wedge e^{4}-e^{2} \wedge e^{3}\right),
$$

The associated potential one-form is given by

$$
\left.A=-\lambda(M+N)\left[-\cos \theta d \varphi+\frac{r-N-\alpha \cos \theta}{\Xi}\left(d t+P_{\theta} d \varphi\right)\right)\right] .
$$


For $\alpha=0$, the above expressions describe self-dual, or anti-self-dual Abelian gauge fields living on the space of a Taub-Nut instanton with an horizon [8]. In the absence of the "magnetic" mass $(N=0)$ we have the gauge fields harbored by the Euclidean-Kerr metric. The latter can also be obtained from the potential one-form in the Kerr-Newman dyon metric after an appropriately Euclideanizing it and setting the electric and magnetic charges equal to each other ( see [25]).

Next, we shall show that these self-dual and anti-self-dual harmonic two-forms are square integrable on the Kerr-Taub-bolt space. This can be shown by explicitly integrating the Maxwell action. For the self-dual two-form we have

$$
\frac{1}{4 \pi^{2}} \int F \wedge F=\frac{\lambda^{2}}{2 \pi^{2}}(M-N) \int_{0}^{t_{0}} d t \int_{0}^{\varphi_{0}} d \varphi=\frac{2 \lambda^{2}}{\kappa}(M-N)\left(1-\frac{2 \alpha}{r_{+}-r_{-}}\right),
$$

where $t_{0}=2 \pi / \kappa$ and $\varphi_{0}=2 \pi(1-\Omega / \kappa)$. Since this integral, which represents the second Chern class $C_{2}$ of the $U(1)$-bundle, is finite, the self-dual two-form $F$ is square integrable on the Kerr-Taub-bolt space. For an anti-self-dual $F$, a plus sign must be introduced between $M$ and $N$ in (18).

It is also useful to calculate the total magnetic flux $\Phi$ which is obtained by integrating the self-dual 2-form $F$ over a closed 2 -sphere $\Sigma$ of infinite radius; dividing this by $2 \pi$ gives the first Chern class with minus sign

$$
-C_{1}=\frac{\Phi}{2 \pi}=\frac{1}{2 \pi} \int_{\Sigma} F=2 \lambda(M-N)\left(1-\frac{2 \alpha}{r_{+}-r_{-}}\right),
$$

which must be equal to an integer $n$ because of the Dirac quantization condition. We see that the periodicity of angular coordinate in the Kerr-Taub-bolt metric affects the magneticcharge quantization rule in a non-linear way. It involves both the "electric" and "magnetic" masses and the "rotation" parameter.

We would like to thank M. J. Perry for helpful discussions.

[1] S. W. Hawking, Phys. Lett. 60A (1977) 81

[2] G. W. Gibbons and S. W. Hawking, Phys. Lett. B 78 (1978) 430

[3] S. T. Yau, Comm. Pure and Appl. Math. 31 (1978) 339

[4] M. F. Atiyah, N. Hitchin and I. M. Singer, Proc. Roy. Soc. A 362 (1978) 425 
[5] N. Hitchin, Polygons and Gravitons, Math. Proc. Camb. Phil. Soc. 85 (1979) 465

[6] G. W. Gibbons and S. W. Hawking, Phys. Rev. D15 (1977) 2752

[7] G.W. Gibbons, S.W. Hawking and M. J. Perry, Nucl. Phys. B138 (1978)141

[8] D. N. Page, Phys. Lett. 78B (1978) 249

[9] G.W. Gibbons and M. J. Perry, Phys. Rev. D22 (1980) 313

[10] Eguchi T and Hanson A J 1978 Phys. Lett. 74B 249

[11] T. Eguchi, P.B. Gilkey and A.J. Hanson, Physics Reports 66 (1980) 213

[12] A. N. Aliev and Y. Nutku, Class. Quant. Grav. 16 (1999) 189

[13] A. N. Aliev, M. Hortacsu, J. Kalayci and Y. Nutku, Class. Quant. Grav. 16 (1999) 631

[14] S. W. Hawking and C. N. Pope, Phys. Lett. 73B (1978) 42

[15] M. J. Duff and J. Madore, Phys. Rev. D18 (1978) 2788

[16] H. Boutaleb-Joutei, A. Chakrabarti and A. Comtet, Phys. Rev. D20 (1979) 1884, Phys. Rev. D20 (1979) 1898, Phys. Rev. D21 (1980) 979, Phys. Rev. D21 (1980) 2280, Phys. Rev. D21 (1979) 2285

[17] J. M. Charap and M. J. Duff, Phys. Lett. 69B (1977) 445, Phys. Lett. 71B (1977) 219

[18] C. Saçlığlu, Class. Quantum Grav. 17 (2000) 485

[19] B. Tekin, Phys. Rev. D 65 (2002) 084035

[20] Sen A, Phys. Lett. B 329 (1994) 217

[21] G. W. Gibbons, Phys. Lett. B 382 (1996) 53

[22] N. Hitchin, Commun. Math. Phys. 211 (2000) 153

[23] G. Etesi, J. Geom. Phys. 37 (2001) 126

[24] A. Papapetrou, Ann. Inst. H. Poincare 4 (1966) 83

[25] B. Carter, in Black Holes, eds. C. De Witt and B. De Witt, (1973) 